\title{
REVIEW \\ Hospital- and community-based interventions enhancing (re) employment for people with spinal cord injury: a systematic review
}

\author{
EH Roels ${ }^{1,2}$, B Aertgeerts ${ }^{3}$, D Ramaekers ${ }^{4}$ and K Peers ${ }^{1,5}$
}

\begin{abstract}
Study design: Systematic Review.
Objectives: To investigate the effect of interventions enhancing (re)employment following spinal cord injury (SCl).

Setting: Studies from multiple countries were included.

Methods: MEDLINE, EMBASE, Cochrane Central Register of Controlled Trials (CENTRAL), CINAHL, PsycINFO and SPORTDISCUS databases were searched. Randomized controlled trials (RCTs) and non-randomized studies (NRSs) describing a hospital- or a community-based intervention aiming at employment in a SCI population were selected. Quality appraisal was done using the SIGN methodology, and the quality of evidence was graded using the Grade approach. Data extraction was performed according to the Cochrane Handbook. Employment rate and duration were primary outcomes.

Results: Only one RCT, including 201 patients describing an intervention over 1 and 2 years, was of sufficient quality. In this study, the employment rate was $26 \%$ after 1 and $31 \%$ after 2 years for competitive work, compared with $10 \%$ in the treatment as usual-intervention site (TAU-IS) control group and $2 \%$ in the treatment as usual observational site (TAU-OS) after 1 and 2 years. Other studies were of low quality and describe higher employment rates from 36 to $100 \%$.

Conclusions: Only one RCT was of sufficient quality and showed evidence that a vocational rehabilitation programme based on the principles of supported employment integrated in a multidisciplinary team enhances employment for SCl people. As the vast majority of studies included in this review are of low methodological quality, further research is needed.
\end{abstract}

Spinal Cord (2016) 54, 2-7; doi:10.1038/sc.2015.133; published online 25 August 2015

\begin{abstract}
INTRODUCTION
Employment is an ultimate and an important goal in the field of rehabilitation medicine, as it adds to quality of life through improving social contacts, self-esteem and financial independence. Particularly in persons with spinal cord injury (SCI), this is important as most persons with SCI are relatively young. SCI unfortunately is a devastating event with a major impact on quality of life. This is not only influenced by the sustained physical disability and impairment but also by the lack of participation in various domains of life among which employment has an important role in community-, social- and civic life activities. ${ }^{1}$ Returning to a productive vocational life has been found beneficial for the physical and mental condition of SCI subjects. $^{2}$ Sir Guttman stated that employment is essential for happiness even if it is not financially necessary. ${ }^{3}$ It has been confirmed that, in case of SCI, employment is related to greater life satisfaction, higher level of activities and better overall health. ${ }^{4}$ Vocational outcome furthermore affects longevity in persons with SCI. ${ }^{5}$ Beside the benefits of employment for the subjects, one would assume benefits for the society. De Vivo et al. ${ }^{6}$ found that indirect costs such as lost wages, benefits, productivity and leisure time often exceed direct costs of SCI,
\end{abstract}

depending on several factors such as age, educational level, level of injury and others. Mackinley et al..$^{7}$ wrote that high unemployment rates in SCI in the USA lead to significant state and federal expenses. These findings emphasize the importance of enhancing vocational reintegration for persons with a SCI.

Employment rates following SCI, however, are low when compared with the normal population. Young et al. ${ }^{8}$ reported employment rates of approximately $40 \%$ (11-56\%) of working age people with a SCI who are more than 12 months post injury. Ottomanelli et al. ${ }^{9}$ describe rates varying from 3 to $80 \%$, with an average rate of $\sim 35 \%$. Lidal et al. ${ }^{10}$ mention employment rates of $21-67 \%$. The results above show a large variation, mainly depending on the methodology used, for example, the terminology and definitions used describing employment (paid/unpaid work, fulltime/part-time work). Nevertheless, employment rates in SCI are low when compared with other chronic illness conditions with intact cognitive functions such as the return to work (RTW) rate in cancer survivors, which is $62 \%{ }^{11}$

Facilitators and barriers associated with employment following SCI include demographic variables (education, sex, race, marital status), injury-related factors (age at injury, level of injury, functional status

${ }^{1}$ Department of Physical and Rehabilitation Medicine, University Hospitals Leuven, Leuven, Belgium; ${ }^{2}$ Department of Rehabilitation Medicine, Center for Rehabilitation, University Medical Center Groningen, University of Groningen, Groningen, The Netherlands; ${ }^{3}$ Department of Public Health and Primary Care, Academic Center of General Practice, KU Leuven, Belgium; ${ }^{4}$ Department of Public Health and Primary Care, Center for Health Services and Nursing Research, KU Leuven, Belgium and ${ }^{5}$ Department of Development and Regeneration, Faculty of Medicine, KU Leuven, Belgium

Correspondence: Dr EH Roels, Department of Rehabilitation Medicine, Center for Rehabilitation, University Medical Center Groningen, University of Groningen, Hanzeplein 1, Postbus 30.001, 9750 RB Groningen, The Netherlands.

E-mail: e.h.roels@umcg.nl

Received 25 June 2014; revised 14 May 2015; accepted 1 July 2015; published online 25 August 2015 
and time since injury), employment history, psychosocial issues (transportation, life satisfaction, locus of control, motivation, expectation and social support) and disability benefit status. ${ }^{9}$ Furthermore, receipt of sickness benefits has also been found to be associated with up to $50 \%$ lower employment rates. ${ }^{12}$

Although employment is a goal of many rehabilitation settings, only few interventions aiming at vocational rehabilitation for SCI have been described in literature. Evidence regarding effectiveness of vocational rehabilitation in SCI is lacking. ${ }^{9}$ Information on and analysis of the characteristics and outcomes of existing interventions enhancing vocational reintegration in SCI are needed to set up a multidisciplinary vocational rehabilitation intervention aiming at a maximal employment rate. A peer reviewed, systematic review of interventions aiming at employment in SCI could offer insight in how to implement an intervention in a hospital and/or a community-based rehabilitation setting and how to organize care after discharge from this setting.

The aim of this study is to identify interventions enhancing employment in the SCI population and report the effect of the intervention on employment rate and duration.

\section{MATERIALS AND METHODS}

\section{Design}

A systematic review looking at studies describing a rehabilitation intervention enhancing employment following SCI was performed. Randomized controlled trials (RCTs) and non-randomized studies (NRSs) such as cohort studies, case series and case reports were included. This implies a higher risk of bias. This was justified, however, to examine the opportunity for undertaking a randomized trial. By providing an explicit evaluation of the weaknesses of available NRS, the design of a subsequent randomized trial can be strengthened.

Only articles written in the English language were withheld. Subjects had to be at least 16 years of age and have suffered a SCI. Exclusion criteria were active and untreated drug or substance abuse and mental impairment affecting safety for self and others.

As primary outcomes, the employment rate and duration of employment were determined. Furthermore, the setting of the intervention being hospital or community-based, the date of start of involvement, frequency and duration of the intervention and the team members involved in the intervention was reported.

\section{Search strategy}

A sensitive search was conducted between February and May 2014. As search terms, both free-text words and subject indexing (MeSH terms) terms were used. No time restriction was used. Further details of the search strategy and the search terms are seen in Figure 1 or are available upon request.

\section{Data sources}

The following electronic databases were searched: MEDLINE, EMBASE, Cochrane Central Register of Controlled Trials (CENTRAL), CINAHL, PsycINFO and SPORTDISCUS.

\section{Selection of studies}

The selection of papers was conducted independently by two reviewers (ER and $\mathrm{BA})$. Disagreements were resolved by referral to a third reviewer (KP). The studies were selected in two phases. At first, title and abstract were screened and potentially relevant documents retrieved. Then, full texts were screened for eligibility, against the inclusion and exclusion criteria described above.

\section{Data extraction and analysis}

Data were extracted by one researcher and checked by a second reviewer, using a data collection form that was designed according to the checklist of items of the Cochrane Handbook. The following data were extracted in the characteristics of included study table: study duration, country, setting, number of participants with SCI, demographics of subjects (age and sex), intervention characteristics (type, start, frequency, duration and team members) and employment situation (previous or new work, paid or unpaid work). As for the interventions, the review covered any kind of intervention aiming at vocational reintegration following SCI. Interventions could be carried out at a hospital and/or a community setting and an in- or outpatient setting. Interventions could primarily focus on different factors such as physical activities, for example, building up strength and endurance (physical interventions), educational activities, for example, teaching activities (educational interventions), environmental adaptations (environmental interventions) or employment activities, for example, workplace adjustments (vocational interventions). Hence, we aimed at dividing interventions into different types: physical interventions, educational interventions, environmental interventions, vocational interventions or multidisciplinary interventions being a combination.

The following outcome data were extracted: percentage of wheelchair bound subjects, employment rate for intervention and the control group and the duration of employment. For potentially high-quality studies, further data were collected by contacting the authors.

As for assessment of study design and quality appraisal, the intention of the study was to use the Cochrane Collaboration's risk of bias assessment tool to assess the main areas of bias being selection bias (random sequence and allocation), performance bias, detection bias, attrition bias, reporting bias and other bias. As the majority of the studies, however, were NRS, the study design for included studies was investigated using the SIGN algorithm for classifying

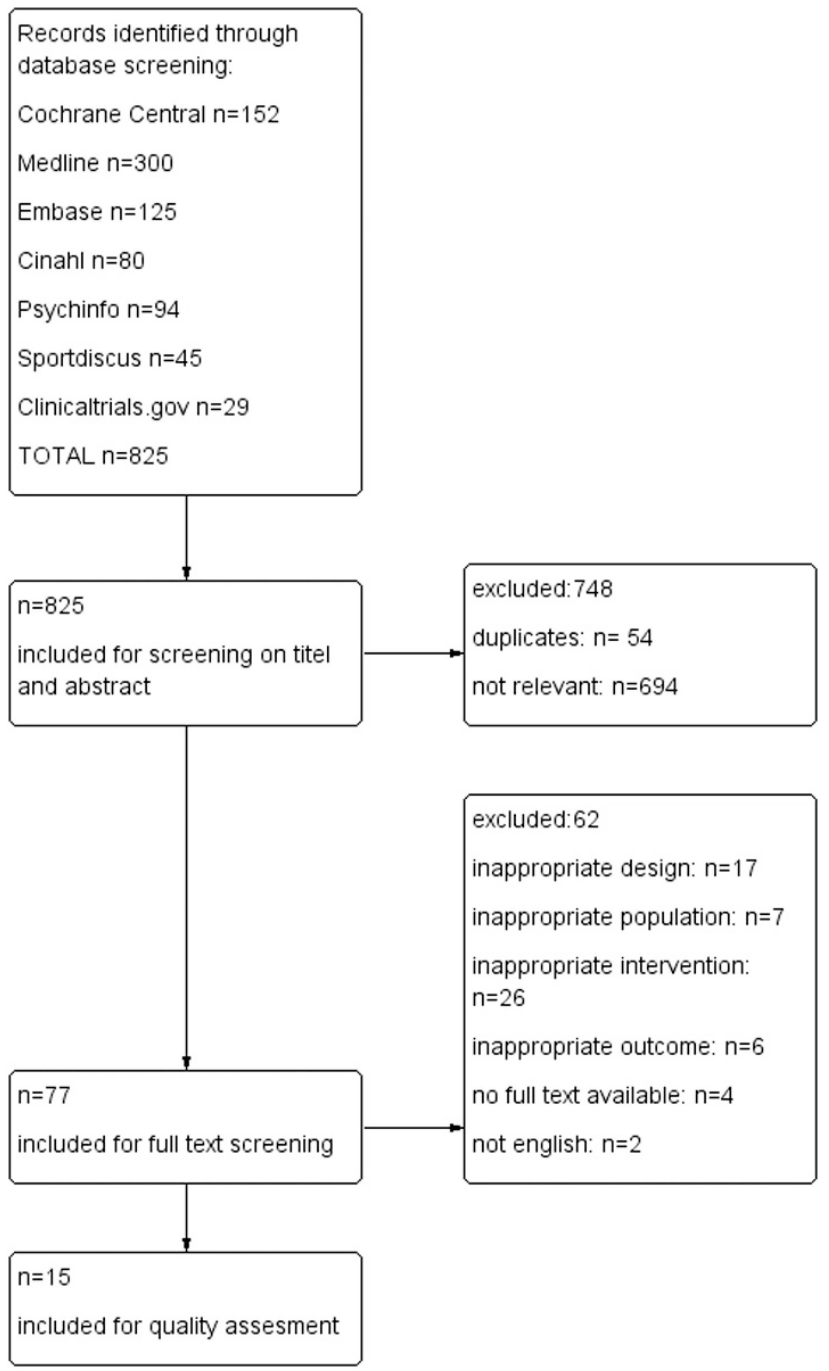

Figure 1 Flow chart of included studies. $n=$ number. 


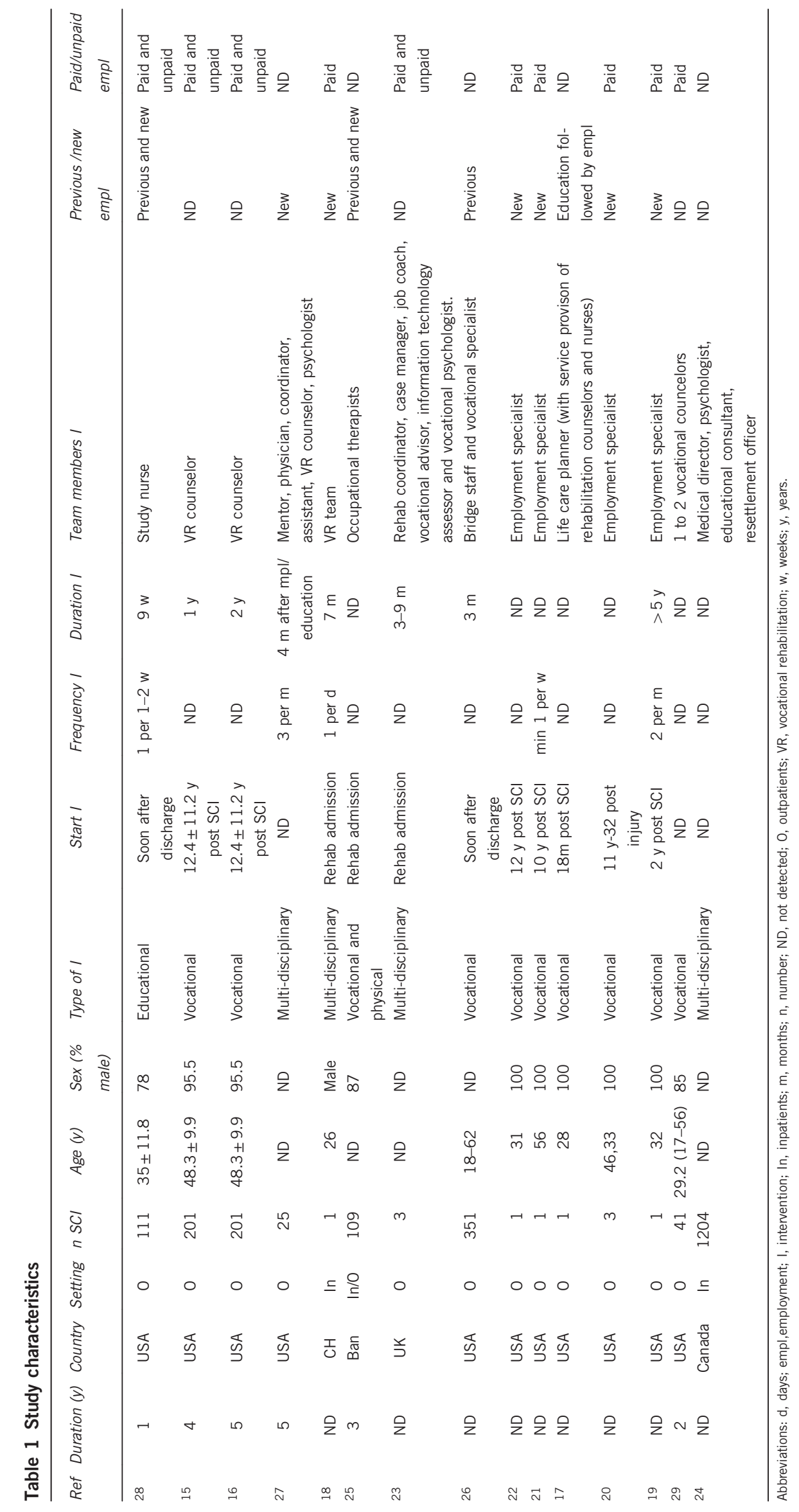




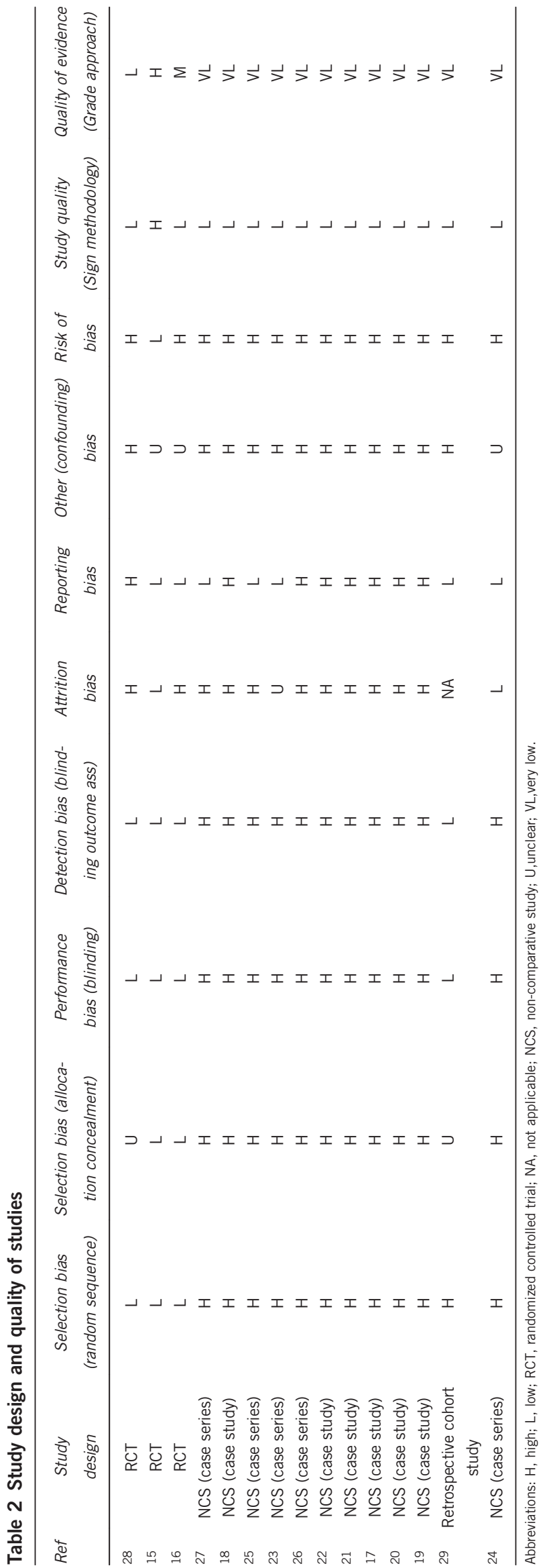

study design for questions of effectiveness. ${ }^{13}$ This methodological assessment is based on a number of key questions that focus on the aspects of study design that research has shown to influence significantly the validity such as randomization, allocation, blinding and reporting. These key questions differ between study types. Each study was thus evaluated for quality using the checklist according to study type. As for case series and case studies, no critical appraisal was performed being aware that the risk of bias is very high. Studies were then rated using the following ranges: high quality (little or no risk of bias), acceptable quality (some risk of bias) and low quality (high risk of bias). The quality of evidence was furthermore graded using the Grade approach. ${ }^{14}$ Studies were rated using the following grades: high, moderate, low or very low quality of evidence.

Because studies were clinically diverse, a meta-analysis was considered meaningless. Also, many studies are presumed to be at a high risk of bias, and therefore results may be seriously misleading.

\section{RESULTS}

Included and excluded studies are shown in Figure 1. Through a comprehensive literature search 825 studies were withheld for screening on abstract and title with the majority being identified from Medline (36\%). After removal of duplicate studies and studies irrelevant to the topic, 77 studies were included for full-text screening. Also studies that did not provide a clear abstract were selected for full-text screening. Out of these studies, 26 (34\%) were excluded not describing a specific work-related intervention, 17 (22\%) having an irrelevant design, 7 (9\%) having an inaccurate population and $6(8 \%)$ not having the pre-specified outcome. For four eligible studies, no full text could be obtained, and two studies were not described in English. Therefore, 15 studies were finally retrieved for inclusion.

Table 1 gives an overview of the main characteristics of the 15 included studies. It is noticeable that two studies describe the same intervention in the same population over 1 and 2 years. ${ }^{15,16}$

Table 2 shows the design and the quality of the included studies. All studies involve a total of 2053 subjects; however, many studies were non-comparative case studies ${ }^{17-22}$ or case series. ${ }^{23-27}$ Only 201 subjects were recruited in a randomized study and 111 subjects in a before/after controlled study. ${ }^{28}$ Even if not all demographic variables were available for all studies, the majority of the participants seem to be male. Eleven out of 15 (73\%) studies were performed in the United States, one in Bangladesh, one in the United Kingdom and one in Canada. Studies were performed at both in- and outpatient settings, as well as a mixed study setting. If studies were performed for outpatients, both community settings as well as an intervention at the subject's house were described. The 15 included studies covered educational, vocational, physical and multidisciplinary interventions (see methods section for explanation of terminology) with the majority of the studies being vocational (67\%), three studies being multidisciplinary, one study being mixed vocational and physical ${ }^{25}$ and one study being educational. ${ }^{28}$ One case $s t u d y{ }^{18}$ was based on the principles of the International Classification of Functioning, Disability and Health (ICF). ${ }^{1}$

As for the start, duration and frequency of the intervention, not all information was available for the included studies. It is noticeable that the time of starting the interventions varies extremely ranging from starting on the first day after sustaining a $\mathrm{SCI}^{18}$ up to a maximum of 34 years after SCI. ${ }^{20}$ Also the frequency of the interventions varies from daily to monthly. The team members of the different interventions are very diverse being either individuals or a multidisciplinary team and either linked to a hospital setting or a community setting.

The majority of the studies, 11 out of $15(73 \%)$, were case reports or case series. Three studies were RCTs. Using the SIGN methodology 
checklist for controlled trials and the Grade approach for quality appraisal, one RCT was of high quality. ${ }^{15}$ One RCT showed moderate and one showed low quality of evidence according to the Grade approach. ${ }^{28,16}$ Further studies are of very low quality of evidence. The judgments of the authors for the main risk of bias groups are also seen in Table 2.

As the majority of studies were of very low quality of evidence using the Grade approach when compared with higher quality studies, results are described separately in different tables below (Tables 3 and 4). The outcomes of the studies with high, moderate or low study quality of evidence using the Grade approach are described in Table 3. Ottomanelli et al. ${ }^{15,16}$ measure the effect of a vocational intervention based on the principles of supported employment on subjects with SCI in two papers. The studies follow a total of 201 cases and the intervention started at an average of 12.4 years post injury. The first study shows the results 1 year after start of the intervention, whereas the second study shows the results 2 years after start of the intervention. In the first study, the employment rate was $26 \%$ for competitive work, comparing with $2.3 \%$ in the treatment as usual observational site control group and $10.5 \%$ in the treatment as usual interventional site control group. The duration of the employment was $17.3 \pm 13.1$ weeks. In the second study, the employment rate was $30.8 \%$ for competitive employment, comparing with $2.3 \%$ in the treatment as usual observational site control group and $10.5 \%$ in the treatment as usual interventional site control group. The duration of the employment rate was $22.2 \pm 14.2$ weeks. It has to be noticed that the attrition rate in the second study of Ottomanelli et al. was high (51\%), which explains the study quality being moderate in contradiction with the first study of the same authors where the study quality was considered high.

In the RCT of Phillips, ${ }^{28}$ an educational intervention aiming at employment was set up; however, data concerning the exact employment rate were lacking, and hence no main conclusions could be drawn.

\section{DISCUSSION}

The objective of this study was to investigate the effect of interventions enhancing (re)employment following SCI.

Only 312 subjects were included in controlled clinical trials. Only one high-quality RCT based on the principles of supported employment and conducted recently in the United States confirmed that a vocational intervention results in an improved employment rate for people with SCI at two points in time. The percentage of employment in this study (26 and $30.8 \%$ ) is still rather low when compared with data mentioned in the introduction $(\sim 30-40 \%)$. This can be explained because the study's sample was largely a chronic SCI population who had been living with both SCI and unemployment for many years, possibly leading to social disenfranchisement

Table 3 Results of high/moderate/low level of evidence studies

\begin{tabular}{|c|c|c|c|c|c|c|c|}
\hline Ref & $n$ I group & n Co group & $n$ Missing & Wheelchair bound \% & Employment rate I group & Employment rate co group & Duration work \\
\hline 28 & $\begin{array}{l}37\left(\begin{array}{l}(1) \\
35(\mid\end{array}\right)\end{array}$ & 39 & 26 & ND & ND & ND & ND \\
\hline 15 & 81 & $\begin{array}{l}76 \text { TAU-IS } \\
44 \text { TAU-OS }\end{array}$ & 25 & $70 \%$ & $\begin{array}{c}29.6 \% \text { All empl } \\
25.9 \% \text { comp empl }\end{array}$ & $\begin{array}{l}\text { 11.8\% TAU-IS all empl } \\
\text { 4.6\% TAU-OS all empl } \\
\text { 10.5\% TAU-IS comp empl } \\
\text { 2.3\%TAU-OS comp empl }\end{array}$ & $17.3 \pm 13.1 \mathrm{w}$ \\
\hline 16 & 81 & $\begin{array}{l}76 \text { TAU-IS } \\
44 \text { TAU-OS }\end{array}$ & 98 & $70 \%$ & $\begin{array}{c}34.6 \% \text { All empl } \\
30.8 \% \text { comp empl }\end{array}$ & $\begin{array}{l}\text { 11.8\% TAU-IS all empl } \\
\text { 4.6\% TAU-OS all empl } \\
\text { 10.5\% TAU-IS comp empl } \\
\text { 2.3\% TAU-OS comp empl }\end{array}$ & $22.2 \pm 14.2 \mathrm{w}$ \\
\hline
\end{tabular}

Abbreviations: comp, competitive; co, control; empl, employment; I, intervention; n, number; ND, not detected; TAU-IS, treatment as usual interventional site; TAU-OS, treatment as usual observational site; w,weeks.

Table 4 Results of very low level of evidence studies

\begin{tabular}{|c|c|c|c|c|c|c|c|}
\hline Ref & n I Group & n Co group & n Missing & Wheelchair bound \% & Employment rate I group & Employment rate co group & Duration work \\
\hline 27 & 25 & 0 & 12 & ND & $36 \%$ & NA & ND \\
\hline 18 & 1 & 0 & 0 & $100 \%$ & $100 \%$ & NA & ND \\
\hline 25 & 46 & 0 & 63 & $17 \%$ & $50 \%$ & NA & ND \\
\hline 23 & 3 & 0 & 13 & ND & $66.70 \%$ & NA & ND \\
\hline 26 & 351 & 0 & 177 & ND & $\begin{array}{l}16.7 \% \text { employment } \\
31.6 \% \text { education }\end{array}$ & NA & ND \\
\hline 22 & 1 & 0 & 0 & $100 \%$ & $100 \%$ & NA & $6 \mathrm{~m}$ \\
\hline 21 & 1 & 0 & 0 & $100 \%$ & $100 \%$ & NA & $6 y$ \\
\hline 17 & 1 & 0 & 0 & $100 \%$ & ND & NA & ND \\
\hline 20 & 3 & 0 & 0 & $100 \%$ & $100 \%$ & NA & ND \\
\hline 19 & 1 & 0 & 0 & $100 \%$ & $100 \%$ & NA & $6 y$ \\
\hline 29 & 20 & 21 & 0 & ND & $75 \%$ & $19 \%$ & ND \\
\hline 24 & 1204 & 0 & 109 & ND & $51 \%$ & NA & ND \\
\hline
\end{tabular}

Abbreviations: comp, competitive; co, control; I, intervention; m, months; $n$, number; ND, not detected; TAU-IS, treatment as usual interventional site; TAU-OS, treatment as usual observational site; w, weeks; y, years. 
and reliance on disability benefits. Another explanation could be the variability in definition of employment. Study employment was defined as competitive employment meaning a paying job earning at least a minimum wage in the community. Volunteer work and sheltered employment did not qualify as employment for the purposes of the authors. Even if medical treatment is similar nowadays, social and cultural care differs among countries, as every country has a different social and insurance system implying different social benefits and vocational care. Therefore, one could assume that the results of Ottomanelli are not directly applicable to other countries. Also, in the study of Ottomanelli, the main group of participants was male, not entirely representing the whole SCI presentation. The general background principles of the intervention are clearly described; however, the exact content and frequency of the intervention are not.

The remaining studies included in our review show better results; however, these studies were of low quality being case studies and case series without the control group, without blinding or randomization implying a very high risk of bias.

A systematic review describing interventions improving employment outcome in SCI was recently published by Trenaman et $a .^{30}$ This review has a similar conclusion as ours stating a profound lack of high level evidence studies focusing on interventions aiming at employment post SCI. However, the objective of the review by Trenaman et al. differed from our review as they primarly aimed at evaluating the intervention itself, whereas in our study we aimed at reporting the effect of the intervention on employment rate and duration of employment. We furthermore performed an extensive search into study quality by critically appraising and grading the included studies. As for results, Trenaman et al. withheld two RCTs. The first RCT being the study of Ottomanelli et al. ${ }^{15}$ that was also withheld in our review. The second RCT suggested that the use of service dogs improves employment outcomes. ${ }^{31}$ This RCT, however, did not fulfill our inclusion criteria, as this intervention was tested on a broad population including SCI next to muscular dystrophy, multiple sclerosis and traumatic brain injury. Results were not analyzed for SCI separately. We were unable to reach the authors of the latter study for more information. Several other studies included in their review did not meet our inclusion criteria mainly because inaccurate or incomplete intervention aiming at employment following SCI. ${ }^{32-35}$

As there is a lack in high-quality studies, we can conclude that further research is warranted and necessary to implement vocational rehabilitation into the care of SCI people.

\section{CONFLICT OF INTEREST}

The authors declare no conflict of interest.

\section{ACKNOWLEDGEMENTS}

We acknowledge the intellectual contribution of Prof Peter Donceel, $\mathrm{PhD}$, Occupational, Environmental and Insurance Medicine, Department of Public Health, KU Leuven, Belgium. Furthermore, special thanks to Dr Victoria Phillips and Dr Lisa Ottomanelli for providing further information.

1 World Health Organisation (WHO). International Classification of Functioning Disability and Health. ICF: Geneva, 2001.

2 Guttman L. Statistical survey on 1000 paraplegic and initial treatment of traumatic paraplegia. Proc R Soc Med 1954; 47: 1099-1113.

3 Guttman L. Our paralyzed fellowmen at work. Rehabilitation 1962; 63: 586-589.
4 Krause JS. Adjustment to life after spinal cord injury: a comparison among three participant groups on employment status. Rehabil Couns Bull 1992; 35: 218-229.

5 Krause JS, Saunders LL, Acuna J. Gainful employment and risk of mortality after spinal cord injury: effects beyond that of demographic, injury and socioeconomic factors. Spinal Cord 2012; 50: 784-788.

6 De Vivo MJ. Causes and costs of spinal cord injury in the United States. Spinal Cord 2013; 35: 809-813.

7 Mackinley W. Assistive technology and computer adaptations for individuals with spinal cord injury. Neuro Rehabil 2004; 19: 141-146.

8 Young AE, Murphy GC. Employment status after spinal cord injury (1992-2005): a review with implications for interpretation, evaluation, further research, and clinical practice. Int J Rehabil Res 2009; 32: 1-11.

9 Ottomanelli L, Lind L. Review of critical factors related to employment after spinal cord injury: implications for research and vocational services. J Spinal Cord Med 2009; 32: 503-531.

10 Lidal IB, Huynh TK, Biering-Sorensen F. Return to work following spinal cord injury: a review. Disabil Rehabil 2007; 29: 1341-1375.

11 Spelten ER, Sprangers MA, Verbeek JH. Factors reported to influence the return to work of cancer survivors: a literature review. Psychooncology 2002; 11: 124-131.

12 Bell SM. The effects of government disability benefits, accessability laws and rehabilitation on employment choices by individuals with spinal cord injuries. ProQuest ETD Collection for FIU. Paper AAI3076642. Available from http://digitalcommons.fiu. edu/dissertations/AAI30766422002.

13 Scottish Intercollegiate Guidelines Network (SIGN). Avalaible from http://www.sign. ac.uk/.

14 Higgins JPT, Green S. Cochrane Handbook for Systematic Reviews of Interventions. Version 5.1.0.2011. Available from http://www.cochrane.org/handbook.

15 Ottomanelli L, Goetz LL, Suris A, McGeough C, Sinnott PL, Toscano R et al. Effectiveness of supported employment for veterans with spinal cord injuries: results from a randomized multisite study. Arch Phys Med Rehabil 2012; 93: 740-747.

16 Ottomanelli L, Barnett SD, Goetz LL. Effectiveness of supported employment for veterans with spinal cord injury: 2-year results. Arch Phys Med Rehabil 2014; 95: 784-790.

17 Blackwell TL, Millington MJ, Guglielmo DE. Vocational aspects of life care planning for people with spinal cord injury. Work 1999; 13: 13-19.

18 Glassel A, Rauch A, Selb M, Emmenegger K, Luckenkemper M, Escorpizo R. A case study on the application of International Classification of Functioning, Disability and Health (ICF)-based tools for vocational rehabilitation in spinal cord injury. Work 2012; 41: 465-474.

19 Inge KJ, Wehman P. Vocational Rehabilitation for persons with Spinal Cord Injuries and other severe Physical Disabilities. Am Rehabil 1996; 22: 2-13.

20 Inge KJ, Wehman P, Strobel W, Powell D, Todd J. Supported employment and assistive technology for persons with spinal cord injury:three illustrations of succesful work supports. J Vocational Rehabil 1998; 10: 141-152.

21 Targett PS, Wehman P, McKinley WO, Young C. Succesful work supports for persons with SCI: Focus on job retention. J Vocational Rehbail 2004; 21: 19-26.

22 Targett P, Wehman P, Young C. Return to work for persons with spinal cord injury: designing work supports. Neuro Rehabil 2004; 19: 131-139.

23 DeSouza M, Sycamore M, Little S, Kirker SG. The Papworth Early Rehabilitation Programme: vocational outcomes. Disabil Rehabil 2007; 29: 671-677.

24 Geisler WO, Jousse AT, Wynne-Jones M. Vocational re-establishment of patients with spinal cord injury. Med Serv J Can 1966; 22: 698-710.

25 Hansen Holm C, Mahmud I, Bhuiyan Jahan A. Vocational reintegration of people with spinal cord lesion in Bangladesh-an observational study based on a vocational training project at CRP. Asia Pacific Disabil Rehabil J 2007; 18: 63-75.

26 King T, Emery R, Warron S, Landis T. A collaboraitive approach to returning clients to work during the first year after spinal cord injury. Top Spinal Cord Inj Rehabil 2004; 9 : 33-42.

27 Kolakowsky-Hayner SA, Wright J, Shem K, Medel R, Duong T. An effective communitybased mentoring program for return to work and school after brain and spinal cord injury. Neuro Rehabil 2012; 31: 63-73.

28 Phillips VL, Hunsaker AE, Florence CS. Return to work and productive activities following a spinal cord injury: the role of income and insurance. Spinal Cord 2012; 50: 623-626.

29 Jellinek HM, Harvey RF. Vocational/educational services in a medical rehabilitation facility: outcomes in spinal cord and brain injured patients. Arch Phys Med Rehabil 1982; 63: 87-88.

30 Trenaman LM, Miller WC, Escorpizo Rthe SCIRE Research Team. Interventions for improving employment outcomes among individuals with spinal cord injury: a systematic review. Spinal Cord 2014; 52: 788-794.

31 Allen K, Blascovich J. The value of service dogs for people with severe ambulatory disabilities. A randomized controlled trial JAMA 1996; 275: 1001-1006.

32 Jongbloed L, Backman C, Forwell SJ, Carpenter C. Employment after spinal cord injury: the impact of government policies in Canada. Work 2007; 29: 145-154.

33 Jang Y, Wang YH, Wang JD. Return to work after spinal cord injury in Taiwan: the contribution of functional independence. Arch Phys Med Rehabil 2005; 86: 681-686.

34 Marini I, Lee GK, Chan F, Chapin MH, Romero MG. Vocational rehabilitation service patterns related to succesful competitive employments outcomes of persons with spinal cord injury. J Vocational Rehabil 2008; 28: 1-13.

35 Wang RY, Yang YR, Yen LL, Lieu FK. Functional ability, perceived exertion and employment of the individuals with spinal cord lesion in Taiwan. Spinal Cord 2002; 40: $69-76$. 